\title{
Volume fraction of the cerebellum in patients with Parkinson Disease
}

\author{
Bunyamin Sahin ${ }^{a}$, Emrah Altunsoy a*, Fikri Ozdemir ${ }^{\mathrm{a}}$, Amani Elfaki ${ }^{\mathrm{a}}$, Ilkay Camlidag ${ }^{\mathrm{b}}$, Meltem Acar Gudek $^{\mathrm{a}}$ \\ ${ }^{a}$ Department of Anatomy, Faculty of Medicine, Ondokuz Mayls University, Samsun, Turkey \\ ${ }^{b}$ Department of Radiology, Faculty of Medicine, Ondokuz Mayıs University, Samsun, Turkey
}

\author{
ARTICLE INFO \\ * Correspondence to: \\ Emrah Altunsoy \\ Deparment of Anatomy, \\ Faculty of Medicine, \\ Ondokuz Mayis University, \\ Samsun, Turkey \\ e-mail: altunsoyemrah@gmail.com
}

\section{Keywords:}

Cerebellum

Magnetic resonance

Parkinson's disease

Planimetry

Volume fraction

\section{ABSTRACT}

Parkinson's disease (PD) is a chronic progressive neurodegenerative disorder, leads to resting tremor, stiffness, slowness and impaired balance. Most investigations on PD focused on the basal ganglia and brainstem, whereas the cerebellum has often been overlooked. Therefore, the aim of this study was determined to describe and compare the cerebral volume and the cerebellar volume and volume fraction in Parkinson's patients comparing with controls. In the present study, the total of 37 subjects, 19 control (9 female, 10 male) and 18 Parkinson's patients ( 8 female, 10 male) were participated. The mean ages of males and females were 54.89, 55.5 and 54.5, 59.2 years-old in controls and patients, respectively. Structural magnetic resonance imaging (MRI) was done to both groups using SIEMENS 1.5 Tesla scanner. The images were analyzed using the ImageJ software. Volume and volume fractions were estimated using the planimetry methods of the stereological techniques. The mean total cerebral volumes were $943.19 \mathrm{~cm}^{3}$ and $909.83 \mathrm{~cm}^{3}$ in control and patient groups, respectively. Even the volumes of the controls were larger than the patients group it did not reached to a statistically significant level ( $\mathrm{p} \geq 0.05$ ). The mean total cerebellar volume and volume fraction were $140.44 \mathrm{~cm}^{3}$, $14.94 \%$ in control and $140.51 \mathrm{~cm}^{3} 15.52 \%$ in patients groups, respectively. No statistically significant differences were observed between the groups $(\mathrm{p} \geq 0.05)$.The findings showed that there was no statistical significant difference between control and patient. There are still controversial opinions about whether the cerebellar volume is affected in PD or not. Our knowledge about cerebellum and PD interaction remains limited, although, the cerebellum is a potential target for symptoms of the PD. Further investigations are needed with higher number of subjects in the groups to understand the changes of the cerebellum in PD.

J. Exp.Clin.Med., 2014; 31:142 